\title{
Simultaneous reconstruction of the oral commissure, lip and buccal mucosa with microvascular transfer of combined first-second toe web and dorsalis pedis flap
}

\author{
Pedro Ciudad ${ }^{1,2}$, Michele Maruccia ${ }^{1,3}$, Stamatis Sapountzis ${ }^{1}$ \& Hung-Chi Chen ${ }^{1}$ \\ 1 Department of Plastic and Reconstructive Surgery, China Medical University Hospital, Taichung, Taiwan \\ 2 Tissue Engineering and Regenerative Medicine Program, National Chung Hsing University, Taichung, Taiwan \\ 3 Department of Surgery 'P.Valdoni', Unit of Plastic and Reconstructive Surgery, University Sapienza of Rome, Rome, Italy
}

\section{Key words}

Oral commissure reconstruction; Lip cancer;

Dorsalis pedis flap; Web space

\section{Correspondence to}

M Maruccia, MD

Department of Plastic and Reconstructive

Surgery

China Medical University Hospital

Taichung

Taiwan

E-mail: marucciam@gmail.com

doi: 10.1111/iwj.12383
Ciudad P, Maruccia M, Sapountzis S, Chen HC. Simultaneous reconstruction of the oral commissure, lip and buccal mucosa with microvascular transfer of combined first-second toe web and dorsalis pedis flap. Int Wound J 2016; 13:787-790

\section{Abstract}

The reconstruction of oral commissure, lip and mucosa defects following tumour resection is a challenging task to the reconstructive surgeon owing to the increasing aesthetic and functional demands. The authors describe a case in which the use of combined first-second toe web with dorsalis pedis flap was transferred and an optimal result was achieved for the oral commissure, lip and buccal mucosa following resection of squamous cell carcinoma and local flap failure.

\section{Introduction}

Selection of the appropriate flap is the key issue for reconstruction of facial defects. The reconstructive approach to defects in the oral commissure, lip and oral mucosa should make every attempt to preserve the function and cosmesis with a minimal amount of morbidity. Oral commissure defect is a particular challenge for reconstruction because the natural morphology of the region is difficult to recreate. There are few tissues in the body that have a morphology similar to that of the oral commissure. Commonly used flaps such as the radial forearm and anterolateral thigh flaps do not recreate this contour well because there is no natural skin crease which lends itself to optimal contouring. Consequently, many patients following oral commissure reconstruction with such flaps have poor aesthetic outcomes, as well as functional deficit, such as drooling from an oral commissure that is round, instead of wedge shaped.

Several methods for the reconstruction of the oral commissure, lip and oral mucosa have been described in the literature ranging from local flaps to free flaps. Local flaps are the mainstays of the reconstruction of the lip-chin complex, but massive defects may need free flap reconstruction.

To our knowledge, this is the first report in which the use of combined first-second toe web with dorsalis pedis flap has achieved an optimal result for oral commissure, lip and buccal mucosa defects.

\section{Case report}

A 47-year-old male with a 6-year history of alcohol, betel nut and cigarette consumption presented a lesion involving oral commissure, adjacent skin and buccal mucosa in the right lower 


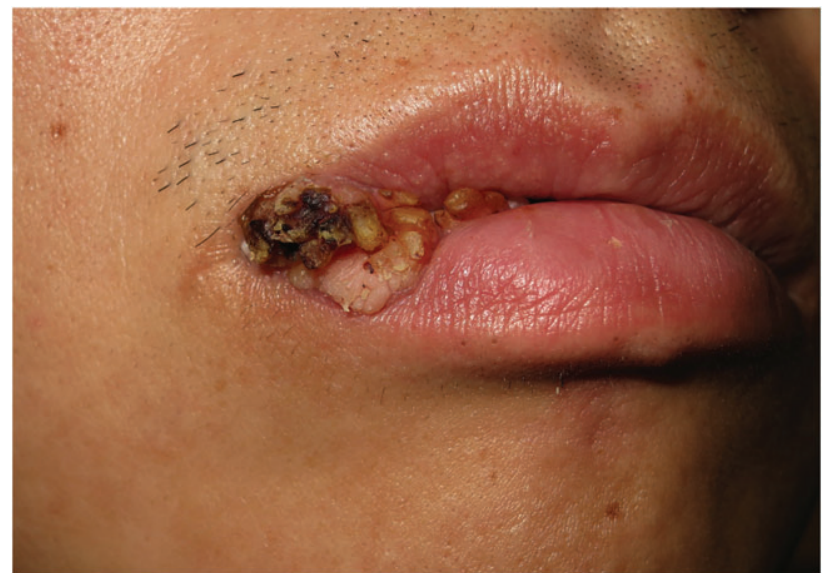

Figure 1 Squamous cell carcinoma of the right oral commissure.

\section{Key Messages}

- reconstruction of the lip commissure with aesthetic and functional fidelity remains a difficult task till today; the goals of reconstruction are symmetry, oral competence and sphincter function for speech and food retention

- full-thickness defects involving oral commissure, lip and buccal mucosa are a challenge for surgeons: several methods ranging from local flaps to free flaps have been described in the literature; for full-thickness defects, local flaps do not provide the aesthetic and functional result, and in these cases free flaps have become an important available option

- simultaneous reconstruction of the oral commissure, buccal mucosa and lower lip can be achieved with combined first-second toe web with dorsalis pedis flap; it is thin, easy to dissect, with a long vascular pedicle and stable blood supply; the size, shape and volume of the flap allowed reconstruction of three-dimensional defects of this anatomical area

lip which gradually increase in size (Figure 1). Clinical examination showed $5-\mathrm{cm}$ verrucous lesion over the right anterior lower lip with involvement of oral commissure and buccal mucosa. A biopsy disclosed squamous cell carcinoma. There was no clinical or radiological evidence of regional or distant metastasis. Because of the extent of the lesion, tumour ablation and reconstruction using local flaps were performed in another hospital. The patient came to our unit after advanced local flap reconstruction with an open wound covered by dermal substitute.(Figure 2) A combined first-second toe web with dorsalis pedis flap was used to resurface the oral commissure, lip and buccal mucosa defect.

\section{Surgical technique}

After the removal of the dermal substitute and toilette of the wound, a defect of $6 \times 3 \mathrm{~cm}^{2}$ in the oral commissure and buccal mucosa as well as a defect of $3.5 \times 3 \mathrm{~cm}^{2}$ in the right lower lip and oral commissure remained.

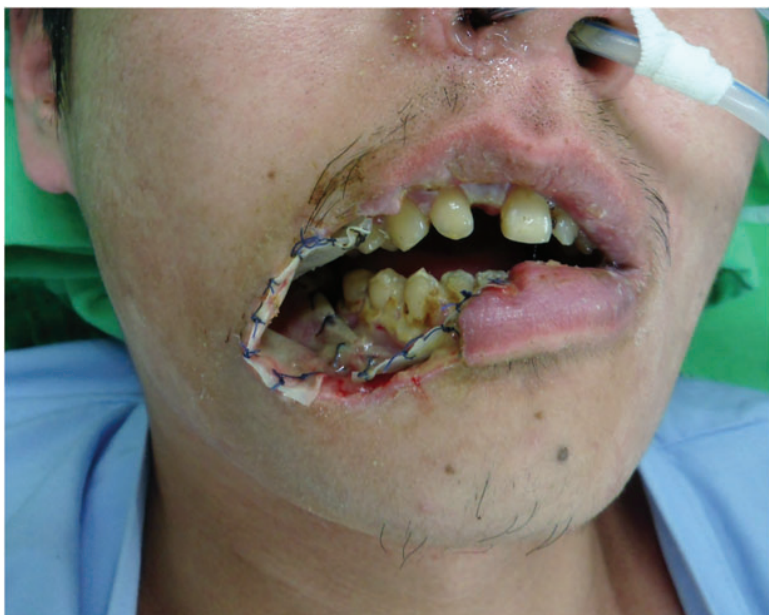

Figure 2 Oral commissure defect after advanced local flap failure covered by dermal substitute.

Preoperatively the dorsalis pedis artery was palpable. Doppler examination confirmed the course of vessels which were marked including the skin of first and second toe web, as well as the dorsalis pedis skin flap (Figure 3A). The flap was raised with the dorsalis pedis artery, one cutaneous vein and one concomitant vein as the vascular pedicle (Figure 3B). Then, the flap was transferred with the following microvascular anastomoses:(i) right anterior facial artery, end to end (ETE) with dorsalis pedis artery, (ii) a branch of right anterior facial vein ETE with dorsalis pedis vein and (iii) another branch of right anterior facial vein ETE with a cutaneous vein of the flap. Inset of the flap was done with the dorsalis pedis skin for the oral mucosa defect, and the toe web flap for the right oral commissure and right lower lip defect. The donor site was covered with split-thickness skin graft. The patient was discharged on the tenth postoperative day.

At 12-month follow-up, the patient had a good contour of the reconstructed oral commissure and with continence (Figure 4). No signs of tumour recurrence were noted. No complications were observed in the donor site (Figure 5).

\section{Discussion}

Oral cancer is one of the most common types of human cancer in the world. Although the risk factors for oral cancer are well recognised in different countries, according to evidence, alcohol, betel nut chewing and cigarette consumption cause oral, nasopharyngeal and related cancers (1).

Aesthetics and function results in the oral commissure and lip must be considered during reconstruction. The ideal reconstruction of such defects should replace external skin; provide adequate mucosal lining and labial sulcus; and maintain adequate buccal aperture (2).

Several methods ranging from local flaps to free flaps for reconstruction of oral commissure and lower lip have been described in the literature. Sometimes, local flaps do not achieve the aesthetic and functional result as in this case. 
Figure 3 (A) Marking of the first-second toe web and dorsalis pedis flap; (B) flap harvested

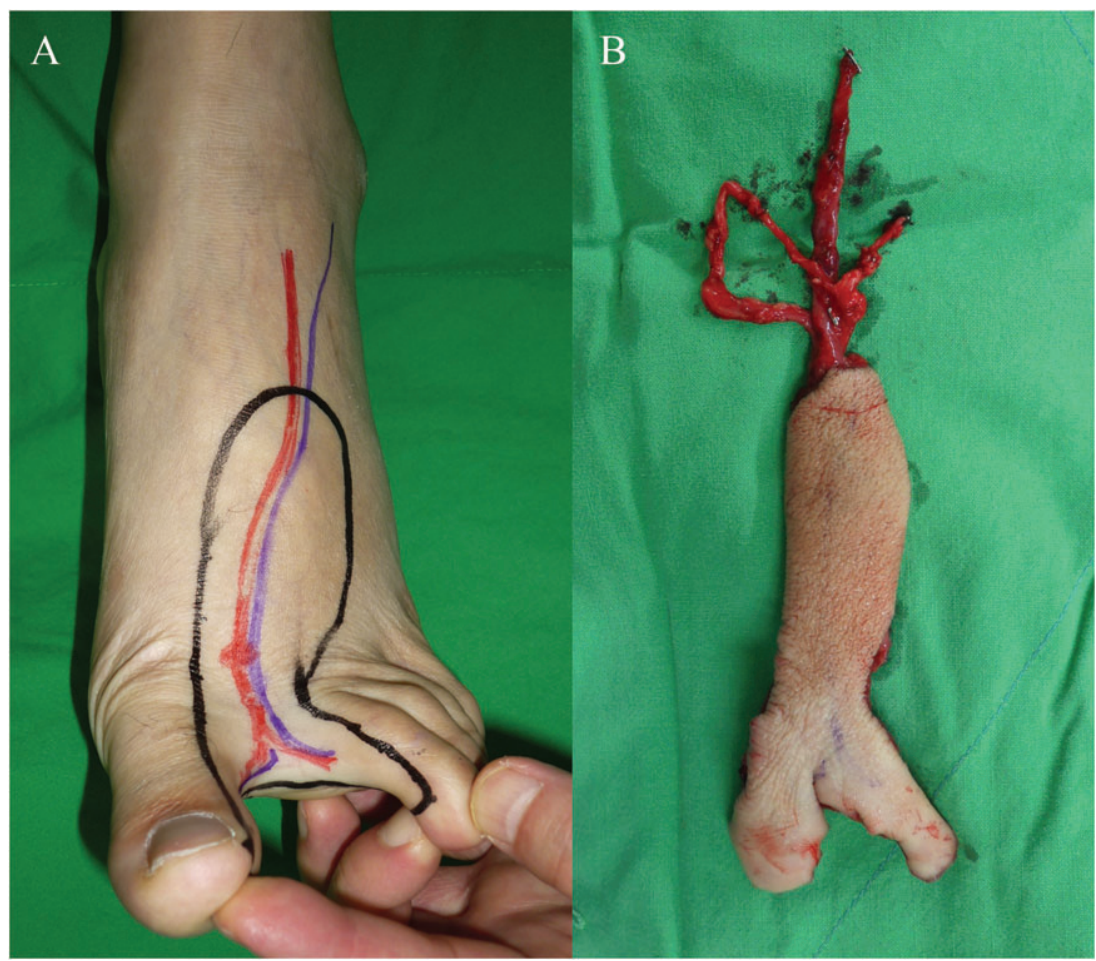

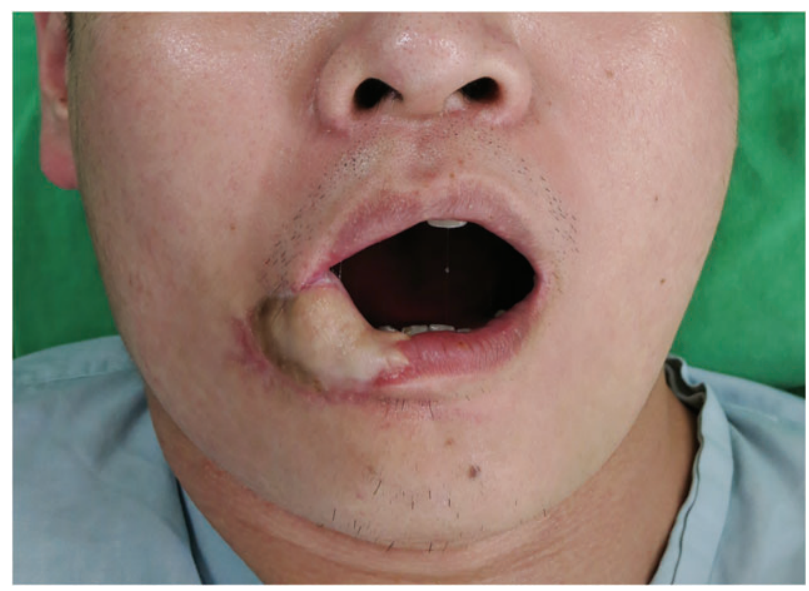

Figure 4 Postoperative view of the patient at 12-month follow-up with good aesthetic and functional result.

With the improvement in microsurgical techniques, free flaps have become an important option available to consider for one-stage reconstruction with good chance of satisfactory aesthetic and functional results (3).

Ohmori and Harii (4) in 1976 introduced the dorsalis pedis flap, and they described their experiences of transferring thin dorsalis pedis flaps in five cases of hand reconstruction. In 1978, Strauch and Tsur (5) were the first to report the use of the first web space, free flap transferred from the foot to the hand. Nowadays, both flaps are used as a free or pedicled fasciocutaneous flap for soft tissue reconstruction of lower-extremity, head, neck and hand defects $(6,7)$.

Sakai et al. (8) introduced the concept of total lower lip and chin reconstruction using a free radial forearm flap, which is

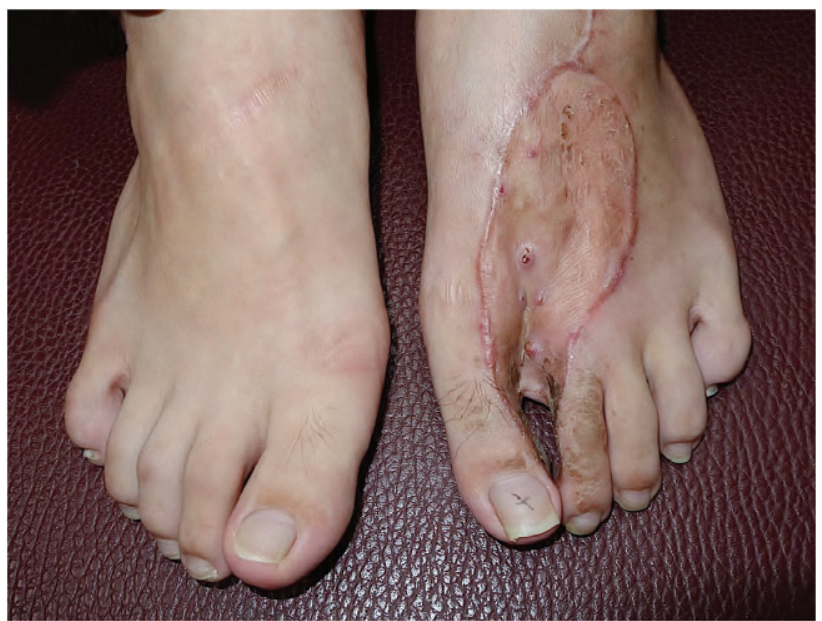

Figure 5 Donor site outcome without complications (12-month follow-up).

considered as a workhorse flap for lip reconstruction because of its pliability and colour match. Radial forearm is frequently used with the tendon of the palmaris longus to support the lip (9). Other free flaps as alternatives for lip and oral commissure reconstructions have been also explored. Yildirim et al. (2) preferred the anterolateral thigh flap because of no sacrifice of a major vessel as in the radial forearm flap and also because of the advantage of primary closure of the donor defect and a hidden donor scar. Koshima et al. (10) reported a combination flap comprising of the pedicled submental flap and the dorsalis pedis flap for the reconstruction of lip and oral commissure. 
However, radial forearm and anterolateral thigh flaps are believed to be bulky for lip and oral commissure reconstruction. The use of two flaps from different regions instead of just one would prolong the operating time and expose the patient to more risk of flap complications.

In this case, we decided to perform a combined first-second toe web with dorsalis pedis flap because it was easy to dissect, thin (thinner than forearm, anterolateral thigh flap or medial sural artery perforator flap), and had a long vascular pedicle and a stable blood supply. Although a potential risk in using this flap was insufficient venous drainage, no problems with blood inflow or outflow were encountered in the present case.

Anatomy of the dorsalis pedis artery is relatively constant and the pulse is usually palpable. We suggest the use of a preoperative Doppler examination to evaluate the blood flow. The cutaneous perforators of the dorsalis pedis artery were located between the base of the first and the second metatarsal bones, and skin paddle was designed according to the location of perforator. The size, shape and volume of the flap were measured according to the individual condition of the defects allowing reconstruction of a three-dimensional defect of the labial commissure. During surgical procedure, care should be taken to preserve the paratenon for the survival of skin grafts at the donor site.

Other advantages of this method are as follows: simultaneous reconstruction of the oral commissure, buccal mucosa and lower lip as a one-stage reconstruction with a single donor site; a good colour match can be obtained; an anatomical structure similar to that of the oral commissure produces excellent results; in cases of head and neck reconstructions, two teams can easily operate simultaneously.

In summary, combined free first-second toe web flap with dorsalis pedis flap are recommended as a good alternative for the reconstruction of the oral commissure, lip and oral mucosa. This technique is one-stage reconstruction that provides reasonable symmetry of the reconstructed area.

\section{Acknowledgements}

All authors hereby declare not to have any potential conflict of interests and not to have received funding for this work from any of the following organisations: National Institutes of Health (NIH); Wellcome Trust; Howard Hughes Medical Institute (HHMI); and other(s). Each author participated sufficiently in the work to take public responsibility for the content.

\section{References}

1. Maruccia M, Onesti MG, Parisi P, Cigna E, Troccola A, Scuderi N. Lip cancer: a 10-year retrospective epidemiological study. Anticancer Res 2012;32:1543-6.
2. Yildirim S, Gideroğlu K, Aydogdu E, Avci G, Akan M, Aköz T. Composite anterolateral thigh-fascia lata flap: a good alternative to radial forearm-palmaris longus flap for total lower lip reconstruction. Plast Reconstr Surg 2006;117:2033-41.

3. Yamauchi M, Yotsuyanagi T, Yokoi K, Urushidate S, Yamashita K, Higuma Y. One-stage reconstruction of a large defect of the lower lip and oral commissure. Br J Plast Surg 2005;58:614-8.

4. Ohmori K, Harii K. Free dorsalis pedis sensory flap to the hand, with microneurovascular anastomoses. Plast Reconstr Surg 1976;58:546-54.

5. Strauch B, Tsur H. Restoration of sensation to the hand by a free neurovascular flap from the first web space of the foot. Plast Reconstr Surg 1978;62:361-7.

6. Ritz M, Mahendru S, Somia N, Pacifico MD. The dorsalis pedis fascial flap. J Reconstr Microsurg 2009;25:313-7.

7. McCraw JB, Furlow LT Jr. The dorsalis pedis arterialized flap: a clinical study. Plast Reconstr Surg 1975;55:177-85.

8. Sakai S, Soeda S, Endo T, Ishii M, Uchiumi E. Compound radial artery forearm flap for the reconstruction of lip and chin defect. Br J Plast Surg 1989;5:337-8.

9. Daya M, Nair V. Free radial forearm flap lip reconstruction: a clinical series and case reports of technical refinements. Ann Plast Surg 2009;62:361-7.

10. Koshima I, Inagawa K, Urushibara K, Moriguchi T. Combined submental flap with toe web for reconstruction of the lip with oral commissure. Br J Plast Surg 2000;53:616-9. 